\title{
Radial current flow and source density in the basal scala tympani
}

\author{
Philip Garcia and Ben M. Clopton \\ Kresge Heturing Research Institute, Lniwersity of Michigan. Ant Amor, Michigat, U.S.4. \\ (Received 16 April 1987; accepted 24 July 1987)
}

\begin{abstract}
Fonic mowement between the scala media and scala tympani is modulated by acoustic stimulation. It underlies electrieal current in the fluids of these compartments and produces voltage gradients from which partial current-flow densities can be estimated. Radial voltage gradients were sampled in the first turn of the scala tympani of the guinea pig cochlea at known distances from the hasilar membranc. Large potential gradients indicated significant current-flow demsities neit the organ of Conti with less llow near the lateral wall of the cochlea and over the spiral lamina. Current-source densities were highest within $100 \mu \mathrm{m}$ of the organ of (orti. Current-source density analysis suggested that source-sink pairs can be detected from field observations in the seala $t \times m p a n$.

Cochlear current: Cochlear microphonic: Cochlea
\end{abstract}

\section{Introduction}

Since its discovery in 1930 by Wever and Bray (1930a, b), the cochlear microphonic (CM) has provided an easily observable indication of the modulation of electrical currents in the cochlea. It has been shown to reverse in phase at the plane of the reticular lamina (Tasaki et al.. 1954), and it is now generally considered to arise from the organ of Corti due to changes in resistivity at the apical ends of the hair cells.

The concept of mechanically induced modulation of resistance at the hair cell, proposed by Davis (1957, 1965), has been expanded with knowledge of cochlear electroanatomy (Strelioff, 1973; Honrubia et al., 1976a; Manley, 1978; Brownell. 1982). At present, an electrical description of the cochlea would include impedance and current-source elements. The current sources are metabolically driven by membrane transport in the stria vascularis (SV) and hair cells of the organ of Corti (Melichar and Syka, 1987). Positive endocochlear potential drives current which is modulated at the hair cells as the stereocilia are bent

Camperpondence to: P. Garcia. Kresge Hearing Research Instrute, Untversity of Michigan. 1301 East Ann. Ann Arbor. MI 48109. U.S.A. and the effective resistance to ionic flow varies (Hudspeth and Corey, 1977), the ionic current probably being carried by potassium ions (Konishi and Salt, 1980). Evidence supporting the hair cells as current modulators as well as the presence of ionic current has been both indirect and direct.

The scala media is lined by cells with tight junctions (Jahnke, 1975; lurato et al.. 1976) and high resistances exist across the chambers of the cochlea (Sitko. 1974). The resistances change with sound stimulation (Strelioff et al., 1972). When the stereociliary bundle is forced toward the taller stereocilia, membrane conductance increases for positively charged ions (Hudspeth and Corey. 1977). Experiments with frog sacculus by Corey and Hudspeth (1979) and isolated vestibular hair cells of chick by Ohmori (1985) suggest that these ionic pores are approximately $0.7 \mathrm{~nm}$ in diameter and readily pass potassium as well as other ions, with preference given to cations. Exposure of the cochlea to kanamycin is selectively toxic to outer hair cells leaving inner hair cells relatively intact as evaluated by light microscope. Dallos (1973a) found that the CM dropped to about $3 \%$ of normal after kanamycin treatment, suggesting that outer hair cells contribute most of the recordable microphonic and thus most of the modulation of ionic current. 
Other than the modulation site at the hair cells and the presumed path through the SV, the magnitudes and paths taken by these currents are uncertain. Direct evidence suggesting the presence of modulated ionic currents has been recently described (Hudspeth, 1982; Brownell et al.. 1983; Zidanic and Brownell, 1986). Hudspeth spatially mapped potentials around isolated hair cells and found maximal response at the apex of the hair bundle, suggesting that ionic flow occurs through the distal tips of the stereocilia. On a larger scale, Brownell et al. (1983) measured acoustically evoked radial current gradients in the third turn of the guinea pig cochlea. Using condensation and rarefaction clicks, he found the radial component to be greatest under the organ of Corti with positive ionic current moving toward and away from the modiolus depending on the polarity of the stimulus.

We investigated radial voltage gradients in a two-dimensional matrix near the basilar membrane (BM) in the first turn of the guinea pig cochlea using tone-burst stimulation. This report describes the techniques used, including their advantages and limitations, as well as examples of current-flow and current-source densities derived from our observations.

\section{Materials and Methods}

\section{Theoretical considerations}

The data consisted of voltage-difference samples, $v(i+1)-v(i)$, which occurred between points $20 \mu \mathrm{m}$ apart along the radial dimension of the basal scala tympani taken medial to lateral. This dimension, $x_{r}$, is a line passing roughly parallel to the BM and bony spiral lamina from the modiolus toward the spiral ligament laterally. The axial and longitudinal dimensions, perpendicular to the BM and parallel to the BM along the direction of the scala tympani, respectively, can be considered orthogonal to the radial dimension on the small scale considered here. Gradients in the axial and longitudinal dimensions were not measured. The voltage differences provide estimates of the radial component of the field gradient and thereby the radial current density:

$$
J\left(x_{\mathrm{r}}\right)=g(x)\left[-\delta v(x) / \delta x_{\mathrm{r}}\right],
$$

under the assumptions that the conductivity $g(x)$ of the perilymph is homogeneous and isotropic. Radial current density as a function of location, $J\left(x_{\mathrm{r}}\right)$. is related to the product of conductivity at that point, $g(x)$, and the negative of the gradient of the potential field along the axis of measurement. $-\delta v(x) / \delta x_{\mathrm{r}}$. The gradient is estimated by the difference operator $\Delta v\left(x_{\mathrm{r}}\right)=[v(1+1)-$ $v(i)] / h$, where $h=20 \mu \mathrm{m}$ is the spatial sampling increment. This should not be confused with the finite-difference equation used for single-ended samples.

The value for the conductivity of perilymph has been estimated to be near that of isotonic saline, $1.42 \mathrm{mS} / \mathrm{mm}$ (Misrahy et al., 1958). In agreement with Brownell et al. (1983), we generally report potential field gradients in preference to current densities because of the uncertainty associated with the exact value of the conductivity of perilymph. Estimates of current densities given here use the conductivity cited.

\section{Preparation}

A total of 45 pigmented guinea pigs weighing 220-350 g were used for these observations. They were initially anesthetized with ketamine hydrochloride (Vetalar, $100 \mathrm{mg} / \mathrm{kg}$ ) and xylazine (Rompun, $5 \mathrm{mg} / \mathrm{kg}$ ) delivered intramuscularly and supplemented regularly to maintain anesthesia. A tracheotomy was performed, and the skull was fixed to a rigid bar with stainless steel screws afixed to the dorsal cranium with dental acrylic to obtain a high degree of stability. A postauricular incision was made and the bulla was opened by shaving the bone with scalpels to minimize acoustic trauma. In most animals the stapedius muscle was cut. The animal was placed on a DC-powered heating pad which maintained core body temperature at $37.5^{\circ} \mathrm{C}$ using feedback from a rectal temperature probe. An additional heating lamp was positioned over the open bulla, and a thermistor probe was placed adjacent to the bulla. The distance of the heating lamp was adjusted to maintain the bulla temperature at $37-38^{\circ} \mathrm{C}$.

When the electrode was to be placed, the round window was incised and partially removed in order to provide access to the scala tympani. The base of the cochlea was chosen because of accessibility, relatively large dimensions, and phase considera- 
tions to be described in the Discussion section. A silver wirc was placed in the neck muscles and acted as the voltage reference for all experiments. From the opening of the cochlea, data collection was generally restricted to $2 \mathrm{~h}$ in order to minimize physiological deterioration (Kelly and Khanna, 1984).

\section{Stimuli}

The stimuli presented were tone bursts having frequencies ranging from 300 to $6000 \mathrm{~Hz}$. They were gated at the same starting phase across presentations. Their rise-fall times were $2 \mathrm{~ms}$, and duration was $40 \mathrm{~ms}$. The interstimulus interval was $140 \mathrm{~ms}$. Presentation was through a Beyer D' -48 earspeaker coupled in a damped. closed system to the external canal after excision of the pinna. The unattenuated peak output of the system was $122 \mathrm{~dB}$ SPL. Most stimuli were presented at 50-40 dB attenuation from maximum. Calibration was accomplished through a computer-automated procedure using a $3 / 8$ inch Brüel and Kjaer condensor microphone sampling the sound field near the tympanic membrane through a calibrated probe tube. The amplitude response varied less than $5 \mathrm{~dB}$ to $6 \mathrm{kHz}$.

\section{Electrodes}

Two types of recording electrodes were used in the experiments. Glass micropipettes were pulled, filled with $3 \mathrm{M} \mathrm{NaCl}$ and beveled to produce tip impedances of 1-5 M $\Omega$. These were used with the first half of the preparations during the development of the experimental technique. These gave reliable DC information which could be used to determine if penetration of the BM had occurred, thus helping to reference the two-dimensional matrix in scala tympani. Their disadvantage was a high common-mode signal between adjacent single-ended samples which compromised the estimation of small gradients.

The data reported here were obtained using differential recording with pairs of teflon-coated, platinum-iridium wires (1-mil Medwire). Two pieces of this wire, which had an uncoated diameter of approximately $25 \mu \mathrm{m}$, were fed through a flame-pulled micropipette with its tip broken to produce a 50-100 $\mu$ II inside diameler. The wires were sealed in place, cut to the same length with a sharp scalpel, and their geometry characterized under a light microscope. Electrodes were selected so that the center-to-center spacing of the cut ends was $50 \mu \mathrm{m}$ with an overall tip dimension of 100 by $50 \mu \mathrm{m}$. This electrode pair was coupled to a high-input-impedance, differential preamplifier and subsequently amplified $5000 \times$ with a bandwidth of $20-25000 \mathrm{~Hz}$. The preamplifier was designed and built in-house so as to have very small bias currents required for metal electrodes.

The analog signal was converted over 1024 sample points with a 12 -bit $\mathrm{A} / \mathrm{D}$ conversion at 25 $\mathrm{kHz}$. Averages of 25 to 100 responses were taken for each voltage sample point in the matrix. After averaging, little high-frequency energy was present in the waveforms minimizing aliasing. Sampling. storage and subsequent calculations were performed with a Nicolet Med-80 computer. The electrodes were moved in a plane determined by the radial and axial dimensions of the cochlea and sampled a planar point matrix roughly perpendicular to the BM. In this matrix, oriented as shown in Fig. 1, the axis of the electrode array coincided with the axial dimension. The potential of the lateral electrode is referred to the medial.

Axial and radial movements were performed by two hydraulic micromanipulators (Trent-Wells) mounted orthogonally. Axial and radial movements, always unidirectional to minimize error due to hysteresis, had an accuracy of $2 \mu \mathrm{m}$. The reversal of hysteresis along these axes was approximately $20 \mu \mathrm{m}$. Radial sampling was performed every $20 \mu \mathrm{m}$ and axial sampling at $25-150$ $\mu \mathrm{m}$ depending on the estimated distance from the $\mathrm{BM}$ and spiral lamina. The potentials were recorded for a radial series (generally medial to lateral). then the electrode was advanced axially, and another radial series was recorded. When the electrode was to be advanced axially, it was positioned radially over the $\mathrm{BM}$ so that if contact with lissue was made during advancement, it would occur at the BM which would facilitate histological reference positioning. A single-ended $C M$ potential recorded at the beginning of each radial series was repeated at the end of each series in order to determine if time varying factors were present. Changes of more than $5 \%$ eliminated data from that series.

Using a fixed electrode near the round window. 


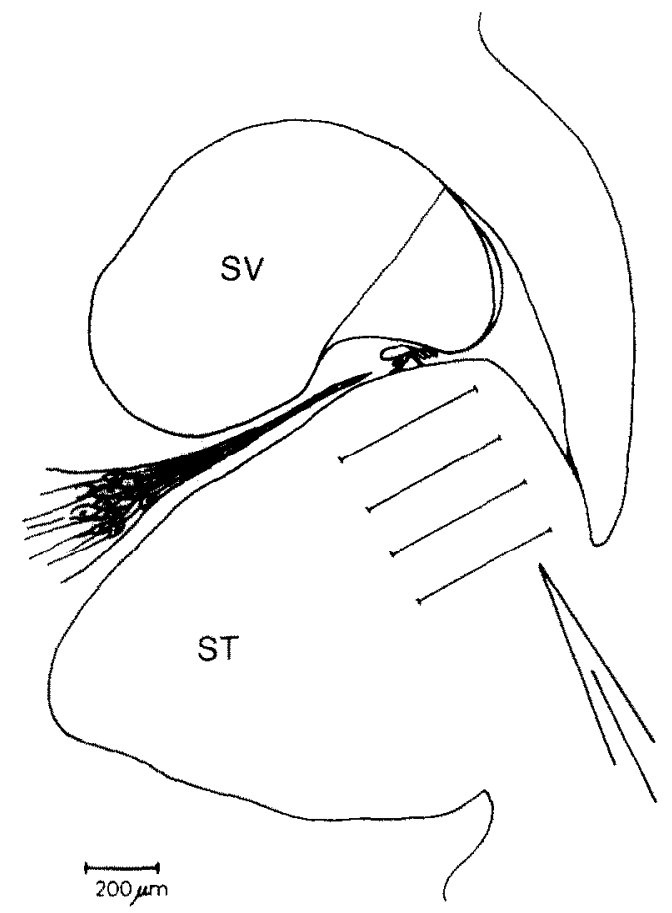

Fig. 1. Radial section of the basal turn of the cochlea through the round window using a camera lucida. The four lines approximately parallel to the organ of Corti represent the radial path recorded by the differential electrode. sampling every $20 \mu \mathrm{m}$. After completing one radial series (line) the electrode was advanced to the next line and another radial series was recorded. This was repeated until the BM was penetrated. SV, scala vestibuli; ST, scala tympani.

acoustically evoked eighth-nerve potentials to rarefaction clicks of $100 \mu \mathrm{s}$ duration were measured in some animals to determine if thresholds would change upon opening the round window. Thresholds for nerve and CM, monitored routinely before and after sampling, varied by less than 3 $\mathrm{dB}$ over the $120 \mathrm{~min}$ required to collect the data that are reported here. Nerve thresholds were less than $40 \mathrm{~dB}$ SPL peak. The CM and eighth-nerve response thresholds were determined by the visual inspection of a waveform averaged over stimulus presentations. Data were usually obtained at 10-20 $\mathrm{dB}$ above threshold where the response was discernible on a single trace of the oscilloscope.

There was some variation in orientation of the matrix from animal to animal. The aim was to maintain radial movement parallel to the basilar membrane. The success of this approach was evaluated by withdrawing the electrode after penetration, moving medially or laterally a fixed distance $(50-100 \mu \mathrm{m})$, and advancing and watching for DC shift (glass electrodes) or other voltage fluctuations to indicate contact. The difference in the penetration depth, after correction for hysteresis, gave an estimate of the angle of the matrix to the membrane as suggested in Fig. 2. Upon penetration of the BM the electrode was removed, the animal overdosed with anesthetic, and the cochlea fixed in $25 \%$ glutaraldehyde by perfusing through the round window after removal of the stapes footplate. The temporal bone was removed postmortem, the cochlea skeletonized. and the BM prepared as a surface preparation. This preparation revealed the location of the $\mathrm{BM}$ penetration which was then referenced to the sampling matrix supporting the visual placement of the electrode. The inclination of the matrix relative to the radial axis of the BM was less than $10^{\circ}$ for all glass electrode trials, but the technique was somewhat unreliable with metal electrodes because of the lack of DC information. Estimates that were obtained from voltage disturbances that were observed agreed with those from the pipette experiments.

\section{Data analysis}

A fast-Fourier transform (FFT) of each radial

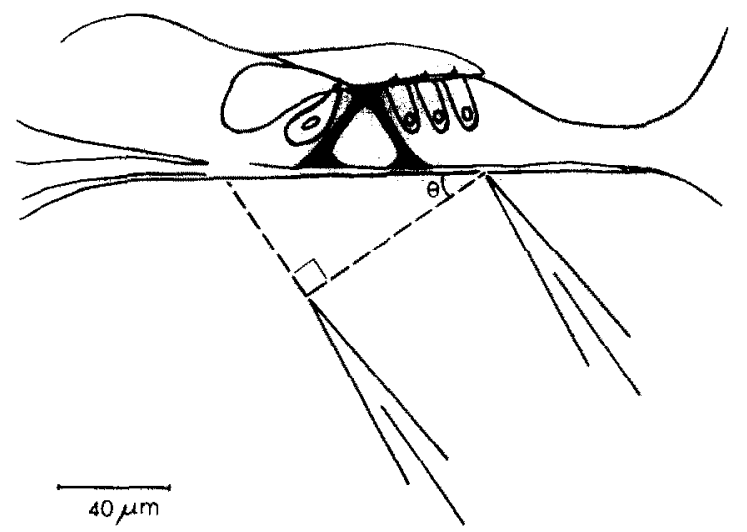

Fig. 2. Illustration demonstrating the technique used to verify matrix position relative to the organ of Corti $(O C)$. After penetration of the $O C$ with change in the $D C$ potential (glass electrodes only) the electrode was withdrawn, moved radially 50 to $100 \mu \mathrm{m}$, and readvanced. By noting the position of the electrode, $\theta$ was determined. It was calculated to be less than $10^{\circ}$ for all successful experiments. 
voltage-gradient average provided magnitude and phase for each spatial sample. The stimulus frequency was chosen to lie at an integer bin value in the FFT in order to optimize the sampling efficiency. These values provided the basis for the descriptions to follow. We observed phase shifts in single-ended $\mathrm{CM}$ records for a number of frequencies in order to obtain phase corrections to estimate the correspondence between $\mathrm{CM}$ polarity and rarefaction or condensation. Phases taken relative 10 the electrical waveform driving the headphone were corrected from such estimates.

The estimation of field gradients from singleended samples along a spatial dimension normally utilizes spatial smoothing and the application of a finite-difference equation (Brownell et al. 1983). Differential recording obviated the second. Spatial smoothing is used for some of the data to be described.

Current-source densities (Nicholson and Freeman, 1975) were obtained by numerical differentiation of a radial series of experimental values. This function, the second spatial derivative of the field along the line of sampling, reflects field divergence, i.e., current entering or leaving the radial dimension of sampling in this case. In a homogeneous medium. this function is maximal nearest current sources and sinks.

\section{Result:}

In the results to be described, we have chosen to focus on the details of data from two animals where the differential metal electrodes were used in order to contrast analysis and display procedures and to show variability. The completeness of the sampling passes near the BM was especially high, and we attach a high confidence to the viability of the preparations, estimation of electrode placement relative to the BM, and representativeness of these data.

Fig. 3 is an example of a differential recording in scala tympani for a $512 \mathrm{~Hz}$ tone burst. The response closely resembles the stimulus waveform and is relatively noise-free after averaging over 50 stimulus presentations. The eighth-nerve compound-action potential, although visible at higher amplifications, is not detectable in the response shown because of trace reduction. The short time

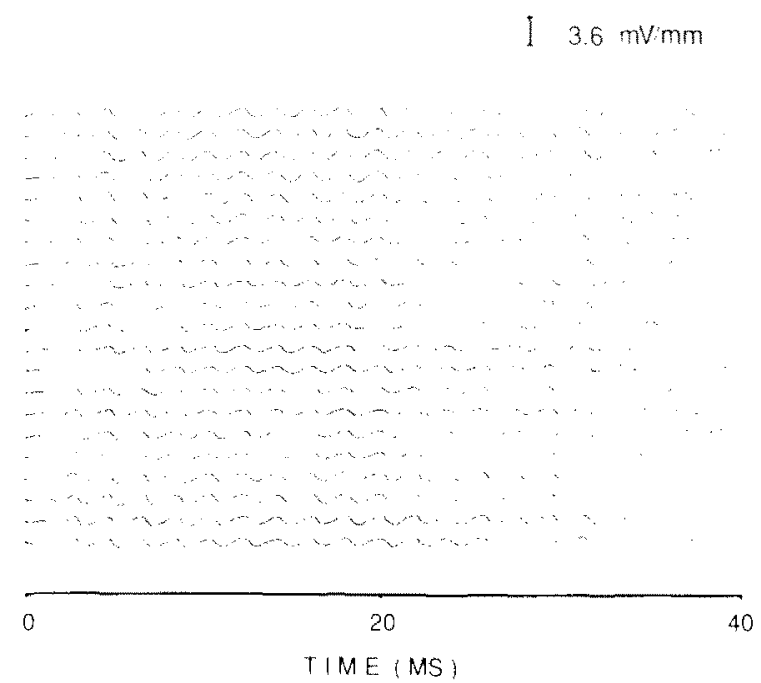

Fig. 3. Example of evoked potentials reorded differentially. Each trace represents an average of 50 stimulus presentations, with the bottom trace recorded medially (over the onseous spiral lamina. and the uppermost trace recorded laterally (near the spiral ligament). The latency of the response is $0.8 \mathrm{~ms}$. Positive is up in all figures and corresponds to the potential at the lateral electrode referenced to the medial and condensation at the tympanic membrane. These correspond to the nearest radial series of Fig. 5 . The intensity was $82 \mathrm{~dB} \mathrm{SPL}$.

delay of the evoked response. $0.8 \mathrm{~ms}$, is indicative of generation in the basal turn and includes mechanical, acoustic, and cochlear components. Phase shifts at different frequencies arose partially from reactive components of the acoustic system and therefore were dependent on frequency and not predictable from a single delay estimate. Radial current, e.g., movement of cations, is indicated to flow medially during pressure condensations and laterally during pressure rarefactions at the tympanic membrane.

We did not observe consistent phase reversals in any of our recordings, either single-ended or differential, over the radial extent of sampling. The phase component of the FIT. referenced to the voltage waveform driving the headphone. varied less than $5^{\circ}$ for most radial series nearest the BM.

Figs. 4 and 5 illustrate the field-magnitude values for five radial series taken in an approach to the $\mathrm{BM}$ in one animal using two stimulation frequencies. The example in Fig. 4 shows one of the more localized peaks observed in radial current 


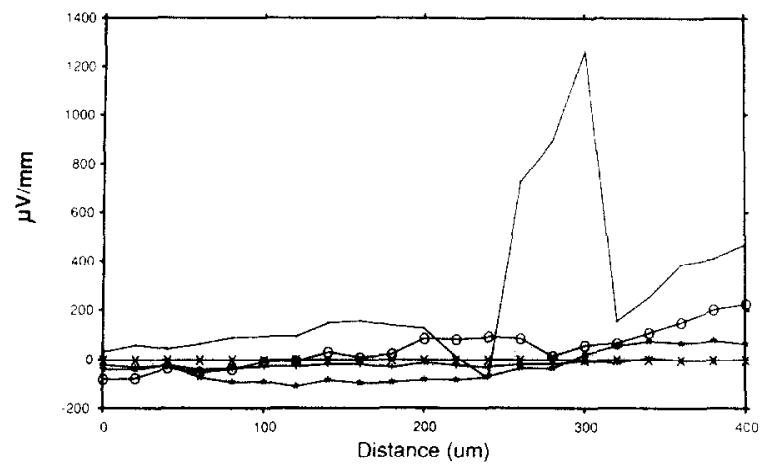

Fig. 4. The magnitude of the field gradient for five radial sequences taken at $20 \mu \mathrm{m}$ steps. The stimulus frequency was 3002 delivered at $82 \mathrm{~dB}$ SPL. The approximated distances from the membrane were $25 \mu \mathrm{m}$ (no symbol), $50 \mu \mathrm{m}$ (O), $100 \mu \mathrm{m}$ $(\star), 200 \mu \mathrm{m}(+)$, and $300 \mu \mathrm{m}(x)$. The sequence taken closes to the BM indicates the presence of especially sharply localized radial current densities. For data analysis, the values for the most distant sequence from $\mathrm{BM}$ were taken as reference.

density with stimulation frequency at $3 \mathrm{kHz}$. This figure also indicates currents especially localized near the BM. It is apparent that closeness to the $\mathrm{BM}$ and spiral lamina was required to observe significant radial densities in this case, generally within $100 \mu \mathrm{m}$ by our estimation. The most distant radial series, invariably lacking large spatial variation, was used as a reference in these illustrations. For some preparations a residual floor on the magnitude at significant distances from putative current sources suggested constant contributions to the recording. Possible sources of this floor will be discussed, but the distant reference excluded it from the data reported here.

Fig. 5 illustrates field magnitudes for a stimulation frequency of $512 \mathrm{~Hz}$ taken at the same sample points as in Fig. 4. The two nearest passes show a minimum in the region of the BM with larger values medially and especially laterally. The maximal values are slightly less than those seen for the higher stimulation frequency, but they were larger at $50 \mu \mathrm{m}$.

The values of Figs. 4 and 5 have the units of $\mu \mathrm{V} / \mathrm{mm}$. They can be converted to current density having units of $\mu \mathrm{A} / \mathrm{mm}^{2}$ with the multiplier 1.42 $\mathrm{mS} / \mathrm{mm}$ as discussed previously. In this example the maximum current density estimate would be $1.8 \mu \mathrm{A} / \mathrm{mm}^{2}$ for the peak in Fig. 4, one of the higher densities obtained.

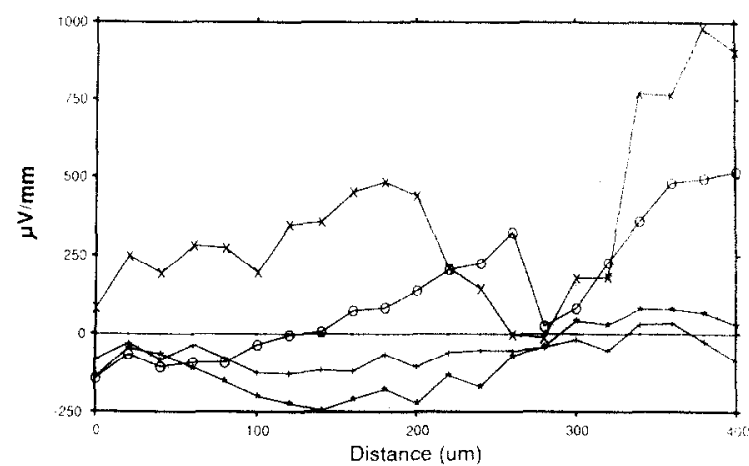

Fig. 5. Field-gradient magnitudes taken at the same loci as in Fig. 4 for a $512 \mathrm{~Hz}$ stimulus. The approximated distances from the membrane were $25 \mu \mathrm{m}(\times), 50 \mu \mathrm{m}(0), 100 \mu \mathrm{m}(\star), 200$ $\mu \mathrm{m}(+)$, and $300 \mu \mathrm{m}(\cdot)$.

Numerical differentiation of the nearest passes in Figs. 4 and 5 is shown in Fig. 6. Positivity in this graph agrees with current contribution to flow in the radial dimension, and negativity indicates radial current loss. As explained in the caption, corrections must be made for differences in phase shift between the two stimulation frequencies. As shown, the curve for the lower frequency corresponds to a condensation phase, and the curve for the higher frequency to a rarefaction phase. This shift relative to the driving voltage at the headphone was due to a resonance near $3 \mathrm{kHz}$ which produced a nonlinear phase change as a function

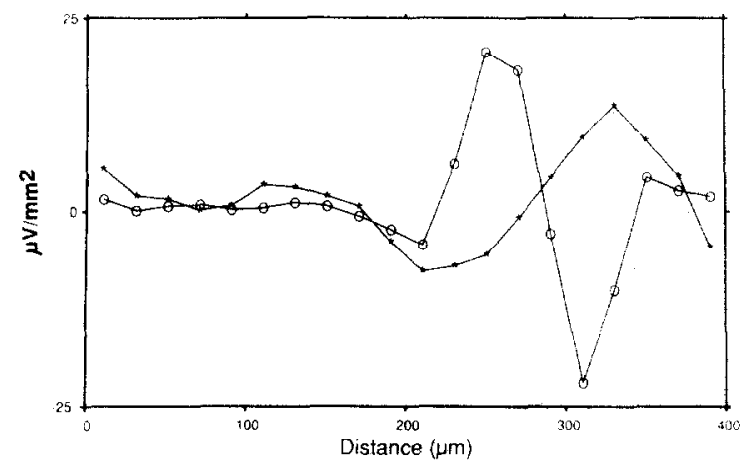

Fig. 6. Current-source density is estimated from the differential of the closest sequences of Figs. $4(0)$ and $5(\star)$ as the second spatial derivative of the field (the first derivative of the field gradients). Positivity suggests current entering the radial axis. After phase correction for travel time to the place of recording for the different stimulus frequencies, the low-frequency data corresponds to condensation at the tympanic membrane and the high-frequency data to rarefaction. Ihree-point spatial smoothing $(0.25,0.5,0.25)$ was used. 
of frequency. After phase correction. these estimates of current-source density indicate current gain laterally and loss medially of radial current during the condensation phase with reversal during rarefaction. This pattern suggests a source-sink pair at about $200-285$ and $285-350 \mu \mathrm{m}$ in the sampling sequence. The extent of the $\mathrm{BM}$ in this experiment was estimated at $285-330 \mu \mathrm{m}$. Assuming that longitudinal currents did not contribute to the observed localized current densities, the alternating source-sink pair can be associated with the BM and adjacent bony spiral lamina in this instance.

Linear interpolation between values of the differential data was used to construct contour plots for radial current-density vectors. Figs. $7-9$ illustrate contours for some radial current-density magnitudes. The gradient contour lines are superimposed on a cross section of the cochlea drawn from experimental material. Error in the superimposition is estimated to be $30-50 \mu \mathrm{m}$, dependent upon reference positioning as well as small variations in electrode placement.

In Fig. 8, for a tone burst at $512 \mathrm{~Hz}$, the greatest radial currents occur in two areas; medial to and at the lateral edge of the organ of Corti. In contrast. as shown in Fig. 7, the higher frequency tone burst evoked the greatest radial current den-

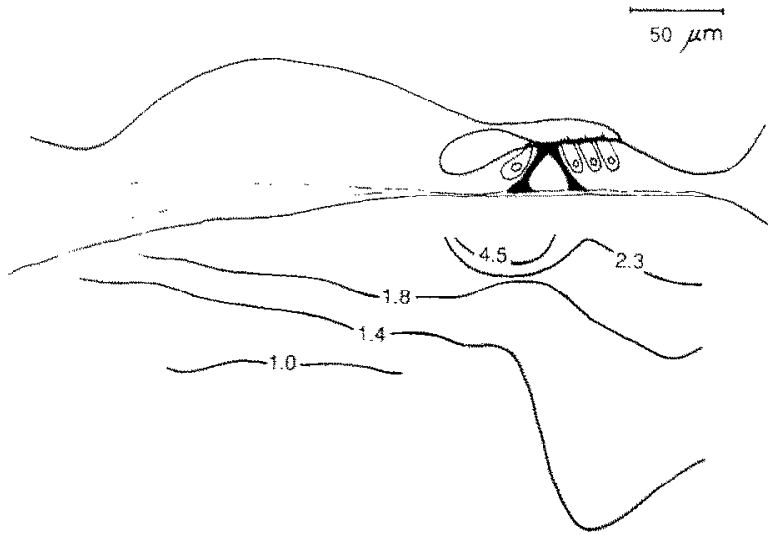

Fig. 7. Interpolation of radial gradients superimposed anatomically over a cross section of the cochlea. Each contour, represented by a line. represents isointensity radial gradients interpolated from a two-dintensional matrix of magnitude de termined from a FFT. Note how more focal gradients are consistenty recorded over the organ of Corti. with significant gradients also present laterally. Stimulus frequency. $3000 \mathrm{~Hz}$ : isointensity contour, $1.0=165 \mathrm{fV} / \mathrm{mm}$ : intensity. $82 \mathrm{~dB}$ SPL.

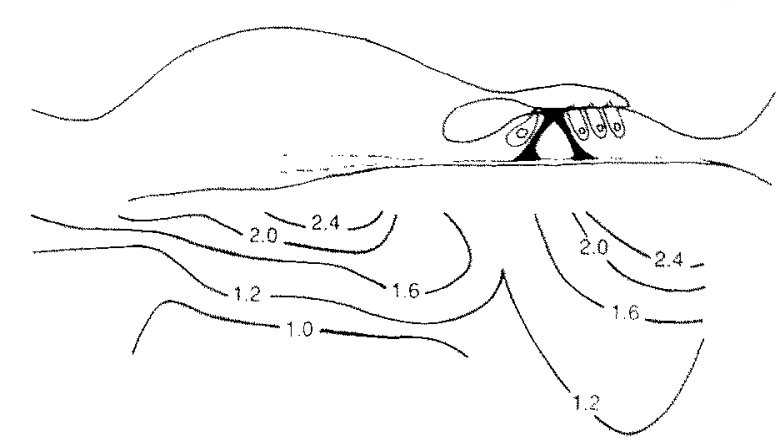

Fig. 8. Isointensity field.gradient contours for a $512 \mathrm{~Hz}$ stimulus. Maximal currents are indicated medially and laterally to the $O C$. Isointensity contour. $1.0=374 \mu \mathrm{V} / \mathrm{mm}$ intensity. 82 dB SIL

sity under the basilar membrane with a significant component laterally. The measurements for the two frequencies were made at the same electrode position in the matrix eliminating placement error as an explanation for the difference in current gradients for the two frequencies.

The estimated current contours were variable enough to make conclusions about the effects of frequency uncertain from our samples. For example. Fig. 9 demonstrates the radial contour for a different animal recorded using a stimulus frequency of $512 \mathrm{~Hz}$. Although the conditions were comparable to those in Fig. 8, a somewhat different contour pattern was seen. Note the presence of significant diffuse radial current flow under the organ of Corti including the spiral lamina medially.

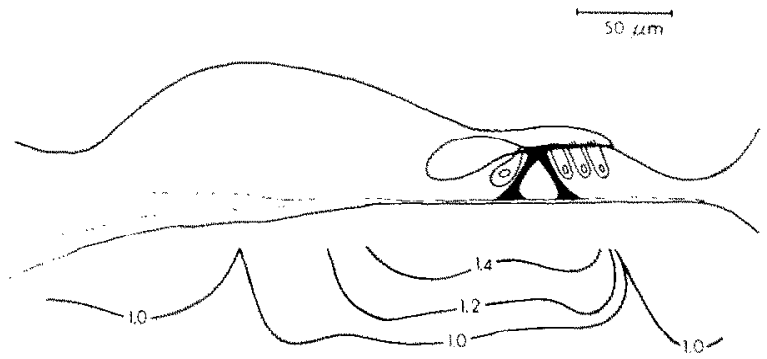

Fig. 9. Isofrequency field-gradient contours, as in Figs. 7 and 8, using data recorded from a different animal. Stimulus frequency. $512 \mathrm{~Hz}$; isointensity contour, $1.0=131 \mu \mathrm{V} / \mathrm{mm}$. Note that the greatest radial gradient is located under the $O C$ as well as medially over the spiral lamina. Intensity, $75 \mathrm{~dB}$ SPL. 


\section{Discussion}

These observations extend those of Brownell et al. (1983) to the basal turn of the cochlea. In addition, they provide measurements of radial current densities for axial displacements from the BM and spiral lamina, and sinusoids were used as stimuli instead of clicks. The magnitude of the radial current densities are of the same order as observed previously, but it was observed that the highest densities lie within approximately $100 \mu \mathrm{m}$ of the plane of the BM and spiral lamina. Current-source density estimates for highly restricted, unidirectional fields support the existence of source-sink pairs in the vicinity of the BM. Since we did not monitor and metal electrodes are not suited for sampling DC potentials, no standing gradients were noted in the absence of acoustical stimulation, so our data provide no evidence about the presence or absence of a current in the absence of stimulation (Brownell, 1982; Zidanic and Brownell, 1986).

Technical and theoretical considerations must qualify the interpretation of these data. The possibility of field distortion caused by the presence of the electrode cannot be ignored for a current source at the site approached by the electrode. The restriction of axial field gradients between the $\mathrm{BM}$ or spiral lamina and the plane of the electrode array of less than $100 \mu \mathrm{m}$ is likely in this case. The primary effect for a nonconductive array surface would be an increase in the observed radial gradients due to the concentration of field lines. Secondarily, since part of the array surface was conductive, capacitive coupling to the metal of the electrode wires with their high conductivity would have its greatest effect at higher frequencies. The second effect appears minimal at the stimulus frequencies used and with the small gradients that exist across the diameter of a $25 \mu \mathrm{m}$ wire. In summary, while ficld distortion was likely, its influence was probably an increase in the observed gradient magnitudes very near the tissue surface at points of significant axial current densities.

A second consideration is the contribution to the recordings of signals not directly linked to radial field gradients. The small, constant gradients measured far from the BM, mentioned earlier. possibly incorporate such components. These were not due to electromagnetic pickup because they disappeared when, as a control, the sound path was blocked. Longitudinal current densities may have contributed to them if the axis of the electrode array was not perfectly radial, a possibility that we cannot exclude. Other possible contributors to baseline values include residual noise or aliasing in the FFT and poor common-mode rejection, but the considerations discussed argue against these. We corrected all data shown here for these factors by vectorial subtraction of the values obtained in the farthest radial sequence where no indication of radial current densities was evident. The current-source density analysis is, of course, unaffected by residual gradients due to its determination from differential and not absolute values of the field gradient.

A third issue is whether disturbing the round window affects the recorded potentials. Evidence from several previous investigators (Békésy, 1960a; Johnstone and Boyle, 1967; Johnstone. 1969: Kohloffel, 1971) indicates that opening the round window and basal turn has a negligible effect. A recent study (Kelly and Khanna, 1984) indicated that histologically, damage was found in the apex and base resembling acoustic trauma. This progressed over time. and became obvious at $16 \mathrm{~h}$ in the cat, with vacuole formation in the afferent nerve and supporting cells. In our experiments, eighth-nerve compound-action-potential thresholds changed less than $3 \mathrm{~dB}$ after opening of the round window. With the above considerations in mind, the duration of the experiments after opening the round window was minimized. generally lasting $2 \mathrm{~h}$ and never exceeding four.

It has been accepted that the inner and outer hair cells are the likely determinants of the CM. and that voltages in the vicinity of the BM represent the sum of a large number of these field generators (Whitfield and Ross, 1965). For a given longitudinal segment of membrane, the amount of phase change per distance is much greater for high frequencies than for low. so that for low frequencies, the CM better represents the individual receptor output (Dallos, 1973b). Kohloffel (1971) looked in detail at Whitfield and Ross's earlier work by simultaneous measurement of potentials in the basal turn of the cochlea, and found that by weighting individual generators' output according 
to the distance from the electrode. CM measurements would correspond to the prediction. Characteristic amplitude minima were found when the measurements were made near the point of maximal stimulation because of out-of-phase summation of generator potentials on either side of the point of maximal stimulation. In order to avoid these phase cancellations, it is necessary to record at a point proximal to the travelling wave peak. It has been pointed out (Honrubia et al., 1976b) that round window measurements will generally record potentials from the basal turn of the cochlea and that because of the phase cancellations mentioned. the CM will be greatly attenuated at frequencies above $8-10 \mathrm{kH}$. Because of these reasons, it was decided to record in the basal turn through the round window.

The radial component of the acoustically evoked potential gradient observed was in one direction for a given stimulus phase indicating that ionic: current flow propagates in one direction along the radial line of sampling for a given hall cycle of a sinusoidal stimulus. Our use of frequencies which were low relative to tuning at the site of recording was intended to minimize phase cancellation of fects and the possibility of longitudinal currents which local longitudinal phase differences would promote. The recording point was located $0.8-1.2$ $\mathrm{mm}$ from the stapes. corresponding to a frequency of $20.50 \mathrm{kHz}$ on the Greenwood (1961) G1 function. All stimuli were 2.5 to 3 octaves below these frequencies.

Through the use of condensation and rarefaction clicks, Brownell et al. (1983) noted the presence of ionic current either toward or away from the modiolus. respectively. which is in agreement with our data. This suggests that the alternating source and sink of the receptor cells modulates these currents which propagate both medially and laterally. Our data do not support a simple alternating dipole since signilicant lateral and medial current vectors were often seen. These suggest maximal radial currents medially over the spiral lamina and near the spiral ligament laterally under some conditions and restricted current loops near the BM in other cases, e.g., between the highand low-frequency conditions of Figs. 4 and 5. Time and spatially varying current patterns are thus indicated. In order to resolve these patterns, it will be necessary to construct a temporal se- quence of spatially defined current vectors over the period of a sinusoid. In addition, stimulus parameters such as frequency and intensity may affect the current patterns. especially in the light of intracellular hair cell recordings which confirm rectification and the filtering of high-frequency waveforms (Russell and Sellick. 1978).

Our data support the previous data of Brownell et al. (1983) and their relationship to kinetic studies (Hudspeth. 1982) which have measured gradient potentials of the order of $0.1 \ldots 7 \mathrm{mV} / \mathrm{mm}$. These gradients indicate currents whose magnitudes we observed to decrease with distance from the plane of the spiral lamina and BM. Significant laterally directed currents medial and lateral to the BM for different stimulus frequencies suggest more complex current patterns than a simple dipole would imply.

\section{Summary}

(1) Radial components of acoustically evoked potentials were found to exist throughout the scala tympani with the greatest gradients occurring near the organ of Corti and smaller gradients near surfaces both medial and lateral to it.

(2) No phase reversal was noted in the gradients nor in the single-ended recordings at any point in the scala tympani of the base of the cochlea. Currents which are primarily laterally directed for rarefactions are indicated.

(3) The phase of the gradients alternates over half cycles of the stimulus indicating ionic movement toward and away from the modiolus.

(4) The voltage gradients and the currents they indicate are consistent with previous studies.

\section{Acknowledgements}

We would like to thank Yolande $\mathrm{Au}$ and Patricia Backoff for their technical assistance. The authors would also like to thank Dr. Lynn Carlisle for her help with anatomical evaluations. This research was funded by grant 85-2090-JI from the Deafness Research Foundation and by grant NS21440 from the NIH.

\section{References}

Békésy. (9. von (1951a) The coarse pattern of the electrical resistance in the cochlea of the guinea pig (xtectrounatomy of the cochlea). I. Acoust. Sox. Am. 23, 18-28. 
Békésy, G. von (1951b) Microphonics produced by touching the cochlear partition with a vibrating electrode. J. Acoust. Soc. Am. 23, 29-35.

Békésy, G. von (1960a) Experiments in hearing. McGraw-Hill. New York.

Békésy, G. von (1960b) In: G.L. Rassmussen and W.F. Windle (Eds.), Neural Mechanisms of the Auditory and Vestibular Systems, CC Thomas, Springfield, IL, p. 92.

Békésy, G. von and Rosenblith, W.A. (1951) The mechanical properties of the ear. In: S.S. Stevens (Ed.), Handbook of Experimental Psychology, Wiley. New York, pp. 1075-1115.

Brownell, W.F. (1982) Cochlear transduction: An integrative model and review. Hear. Res. 6, 335-360.

Brownell, W.E., Manis, P.B., Zidanic, M. and Spirou, G.A. (1983) Acoustically evoked radial current densities in scala tympani. J. Acoust. Soc. Am. 74, 792 800.

Corey, D.P. and Hudspeth, A.J. (1979) Ionic basis of the receptor potential in a vertebrate hair cell. Nature 281. $675-677$.

Dallos, P. (1973a) Cochlear potentials and cochlear mechanics. In: A. Moller (Ed.), Basic Mechanisms in Hearing. Academic Press, New York, pp. 335-372.

Dallos, P. (1973b) The Auditory Periphery. Academic Press, New York.

Davis, H. (1957) Biophysics and physiology of the inner ear. Physiol. Rev. 37, 1-49.

Davis, H. (1965) A model for transducer action in the cochlea. Cold Spring Harbor Symp. Quant. Biol. 30, 181-190.

Greenwood, D.D. (1961) Critical bandwidth and the frequency co-ordinates of the basilar membrane. J. Acoust. Soc. Am. $33,1344-1356$.

Honrubia, V., Strelioff, D. and Sitko, S.T. (1976a) Physiological basis of cochlear transduction and sensitivity. Ann. Otol. Rhinol. Laryngol. 85, 697-711.

Honrubia, V., Strelioff, D. and Sitko, S.T. (1976b) Electroanatomy of the cochlea: Its role in cochlear potential measurements. In: R.J. Rubel, C. Elberling and G. Salomon (Eds.), Electrocochleography, University Park Press.

Hudspeth, A.J. (1982) Extracellular current flow and the site of transduction by vertebrate hair cells. J. Neurosci. 2, 1-10.

Hudspeth, A.J. and Corey, D.P. (1977) Sensitivity, polanty, and conduction change in the response of vertebrate hair cells to controlled mechanical stimuli. Proc. Nat. Acad. Sci. USA 74, 2407-2411.

Iurato, S., Franke, K., Luciano, L., Wermbter, G., Pannese, E. and Reale, $F_{n}(1976)$ Fracture faces of the junctional complexes in the reticular membrane of the organ of Corti. Acta Oto-Laryngol. 81, 36-47.

Jahnke, K. (1975) The finc structure of freeze-fractured intercellular junctions in the guinea pig inner ear. Acta OtoLaryngol. Suppl. 336, 1-40.

Johnstone, B.M. (1969) Mechanical aspects of cochlear function. In: R. Plomp and R.F. Smoorenburg (Eds.), Driebergen Symposium, Sijthoff, Leiden, The Netherlands.

Johnstone, B.M. and Boyle, A.J.F. (1967) Basilar membrane vibration examined with the Mossbauer technique. Science $158,389-390$.

Johnstone, B.M., Johnstone, J.R, and Pugsiey, I.D. (1966) Membrane resistance in endolymphatic walls of the first turn of the guinea pig cochlea. J. Acoust. 50 . Am. 23, $29-35$.

Kelly, I.P. and Khanna, S.M. (1984) Distribution of cochlear damage caused by the removal of the round window membrane. Hear. Res. 16, 109-126.

Kohtoffel, L.U.E. (1971) Studies of the distribution of cochlear potentials along the basilar membrane. Acta Otolaryngol. Suppl. 288, $1-66$.

Konishi. T. and Salt. A.N. (1980) Permeability of potassium to the endolymph-perilymph barrier and its possible relation to hair cell function. Exp. Brain Res. 40, 457-463.

Manley. G.A. (1978) Cochlear frequency sharpening -- a new synthesis. Acta Otolaryngol. 85, 167-176.

Melichar, 1. and Syka. J. (1987) Electrophysiological measurements of the stria vascularis potentials in vivo. Hear. Res. $25,35-43$.

Misrahy, G.A., Hildreth, K.M., Shinabarger, EW and Gannon. W.J. (1958) Electrical properties of wall of endolymphatic space of the cochlea (guinea pig). Am. J. Physiol. 194, 396-402.

Nicholson, C. and Freeman, A.A. (1975) Theory of current source-density analysis and determinations of conductivity tensor for anuran cerebellum, J. Neurophysiol. $38,356-368$.

Ohmori. H. (1985) Mechano-electrical transduction currents in isolated vestibular hair cells of the chick. J. Physiol. 359. 189-217.

Russcll, 1.J. and Scllick, P.M. (1978) Intracellular studies of hair cells in the mammalian cochlea. J. Physiol. 284. $261-290$.

Sitko. S. (1974) Electrical network properties of the guinea pig cochlea. PhD Thesis, University of California at San Diego.

Strelioff. D. (1973) A computer simulation of the generation and distribution of cochlear potentials. J. Acoust. Soc. Am. $54,620-629$.

Strelioff, D., Haas, G. and Honrubia, V, (1972) Sound-induced electrical impedance changes in the guinea-pig cochlea. $J$. Acoust, Soc. Am. 51, 617-620.

Tasaki. 1. (1954) Nerve impulses in individual auditory nerve fibers of guinea pig. J. Neurophysiol. 17, 97-122.

Tasaki, 1., Davis, H. and Legouix, J.P. (1952) The space-time pattern of the cochlear microphonics (guinea pig), as recorded by differential electrodes. J. Acoust. Snc. Am. 24, $502-518$.

Tasaki, 1., Davis, H. and Eldredge, D.H. (1954) Exploration of cochlear potentials with a microelectrode. J. Acoust. Soc. Am. 26, 765-773.

Wever, E.G. and Bray, C.W. (1930a) Auditory nerve impulses. Science $71,215$.

Wever, E.G, and Bray, C.W. (1930b) Action currents in the auditory nerve in response to acoustical stimulation. Proc. Natl. Acad. Sci. USA 16, 344-350.

Whitfield, I.C. and Ross, H.F. (1965) Cochlear microphonic and summating potentials and the outputs of individual hair cell generators. J. Acoust. Soc. Am. 38, 126-131.

Zidanic. M. and Brownell, W.E. (1986) Cochlear hair cell currents revealed by potential gradient analysis of microphonic potentials in scala tympani. Abstr. Assoc. Res. Otolaryngol. 9.61. 